\title{
Altered Actin Filament Dynamics in the Drosophila Mushroom Bodies Lead to Fast Acquisition of Alcohol Consumption Preference
}

\author{
Andrew R. Butts, ${ }^{1 \star}$ Shamsideen A. Ojelade, ${ }^{6 *}$ Eva D. Pronovost, ${ }^{1}$ Alexandra Seguin, ${ }^{1}$ Collin B. Merrill, ${ }^{1}$ \\ (D)Aylin R. Rodan, ${ }^{1,2,3}$ and (DAdrian Rothenfluh ${ }^{1,3,4,5}$ \\ ${ }^{1}$ Molecular Medicine Program, 2 ${ }^{2}$ epartment of Internal Medicine, Division of Nephrology, and Departments of ${ }^{3}$ Human Genetics, ${ }^{4}$ Psychiatry, \\ ${ }^{5}$ Neurobiology \& Anatomy, University of Utah, Salt Lake City, Utah 84112, and ${ }^{6}$ Department of Psychiatry and Neuroscience Graduate Program, University \\ of Texas Southwestern Medical Center, Dallas, Texas 75390
}

Alcohol use is highly prevalent in the United States and across the world, and every year millions of people suffer from alcohol use disorders (AUDs). Although the genetic contribution to developing AUDs is estimated to be $50-60 \%$, many of the underlying molecular mechanisms remain unclear. Previous studies from our laboratory revealed that Drosophila melanogaster lacking RhoGAP18B and Ras Suppressor 1 (Rsu1) display reduced sensitivity to ethanol-induced sedation. Both Rsul and RhoGAP18B are negative regulators of the small Rho-family GTPase, Rac1, a modulator of actin dynamics. Here we investigate the role of Racl and its downstream target, the actin-severing protein cofilin, in alcohol consumption preference. We show that these two regulators of actin dynamics can alter male experience-dependent alcohol preference in a bidirectional manner: expressing either activated Racl or dominant-negative cofilin in the mushroom bodies (MBs) abolishes experience-dependent alcohol preference. Conversely, dominant-negative Racl or activated cofilin MB expression lead to faster acquisition of alcohol preference. Our data show that Racl and cofilin activity are key to determining the rate of acquisition of alcohol preference, revealing a critical role of actin dynamics regulation in the development of voluntary selfadministration in Drosophila.

Key words: addiction; alcohol; Drosophila; genetics

\section{Significance Statement}

The risks for developing an alcohol use disorder (AUD) are strongly determined by genetic factors. Understanding the genes and molecular mechanisms that contribute to that risk is therefore a necessary first step for the development of targeted therapeutic intervention. Here we show that regulators of actin cytoskeleton dynamics can bidirectionally determine the acquisition rate of alcohol self-administration, highlighting this process as a key mechanism contributing to the risk of AUD development.

\section{Introduction}

Alcohol use is highly prevalent; in the United States, $56 \%$ of adults reported they were monthly drinkers, and in 2015, 15 million adults were diagnosed with alcohol use disorder (AUD). The

\footnotetext{
Received April 30, 2019; revised Aug. 22, 2019; accepted Sept. 13, 2019.

Author contributions: A.R.B., S.A.O., A.R.R., and A.R. designed research; A.R.B., S.A.O., and E.D.P. performed research; A.R.B., S.A.O., A.S., and C.B.M. contributed unpublished reagents/analytic tools; A.R.B., S.A.O., E.D.P., and A.R. analyzed data; A.R.B., S.A.O., A.S., C.B.M., A.R.R., and A.R. wrote the paper.

This work was supported by NIH T32DA007290, F31AA021340 (S.A.0.), AHA 16CSA28530002, NIH R01DK110358 (A.R.R.), NIH R01AA019526 (A.R.), U2M2 (A.R.R., A.R.), and the University of Utah Neuroscience Initiative (A.R.). We thank laboratory members for continued discussion of experiments and suggestions on the paper.

The authors declare no competing financial interests.

*A.R.B. and S.A.O. contributed equally to this work.

Correspondence should be addressed to Adrian Rothenfluh at Adrian.Rothenfluh@hsc.utah.edu.

https://doi.org/10.1523/JNEUROSCI.0973-19.2019

Copyright $\odot 2019$ the authors
}

misuse of alcohol results in 88,000 annual deaths and in a \$249 billion economic burden (Centers for Disease Control and Prevention, 2014; Sacks et al., 2015; Substance Abuse and Mental Health Services Administration, 2015). Up to $60 \%$ of the risk of developing AUD can be attributed to genetic predisposition (Dick et al., 2006; Gelernter and Kranzler, 2010), and numerous genes are associated with human AUD phenotypes in genomewide association studies. However, the genes from these association studies often do not group into obvious overrepresented categories of physiological function (Evangelou et al., 2019), leaving many of the molecular pathways of AUD obscure.

Numerous model organisms have been established to investigate these genetic mechanisms. Drosophila melanogaster, the vinegar fly, is one genetically tractable model organism that has been used to model addiction-like behaviors (Rodan and Rothenfluh, 
2010; Kaun et al., 2012). Flies naïvely respond to alcohol similarly to humans: low doses result in loss of inhibition and hyperactivity, whereas high doses result in loss of postural control and sedation (Wolf et al., 2002; Lee et al., 2008). Flies will also learn to prefer alcohol and voluntarily choose to consume it over a period of $5 \mathrm{~d}$ (Devineni and Heberlein, 2009). Initially, flies display naive aversion to ethanol, but a pre-exposure to vaporized alcohol leads to preferential ethanol self-administration (Peru y Colón de Portugal et al., 2014). Numerous genes whose mutations cause altered self-administration in Drosophila also have human orthologs with polymorphisms that associate with AUD phenotypes (Gonzalez et al., 2018; Grotewiel and Bettinger, 2015; Ojelade et al., 2015b).

One of the pathways linked to behavioral responses to alcohol is the dynamic regulation of the actin cytoskeleton, which centers around the Rho-family of small GTPases (Rothenfluh and Cowan, 2013). Loss of RhoGAP18B in flies, a negative regulator of Rac1 GTPase, leads to changes in ethanol-induced sedation or hyperactivity, depending on the RhoGAP18B isoform affected (Rothenfluh et al., 2006). Loss of a second negative regulator of Rac1, Rsu1, also causes altered sensitivity to ethanol-induced sedation, as well as altered alcohol self-administration in a $5 \mathrm{~d}$ preference assay (Ojelade et al., 2015b). Here, we investigate the role of Rac1, and its downstream mediator, cofilin, an actinsevering protein, in experience-dependent alcohol preference (EDAP). Using a behavioral paradigm that separates an alcohol pre-exposure from the consummatory choice, we show that proper regulation of actin dynamics is required during the acquisition for preference to develop. In a new preference paradigm that allows close temporal observation of consummatory behavior, we then show that genetic manipulation that causes the opposite effect on actin dynamics leads to an accelerated acquisition of alcohol consumption preference. These bidirectional phenotypes emphasize the critical role regulators of actin-dynamics play in voluntary alcohol self-administration.

\section{Materials and Methods}

Fly stocks and genetics. Male flies were used for all experiments. Flies were grown and kept on standard cornmeal/agar medium at $25^{\circ} \mathrm{C}$ with $70 \%$ relative humidity. Male $w^{*}$ Berlin flies were used as controls. Transgenic flies were outcrossed to the $w^{\star}$ Berlin genetic background for at least five generations, with the exception of $M B$-GeneSwitch, for which siblingmatched controls were used to equalize their genetic background in our behavioral assays. Transgenic flies were obtained from the Bloomington Stock Center: UAS-Rac ${ }^{C A}$ (RRID:FlyBase_FBst0006291), UAS-Rac ${ }^{D N}$ (RRID:FlyBase_FBst0006290), UAS-tsr ${ }^{C A}$ (RRID:FlyBase_FBst0009236), $U A S$ - $t s r^{D N}$ (RRID:FlyBase_FBst0009238). MB-GeneSwitch (Osterwalder et al., 2001; RRID:FlyBase_FBst0081013) and MB247-Gal4 (Zars et al., 2000; RRID:FlyBase_FBst0050742) were gifts from Dr. Gregg Roman, University of Mississippi.

Drug feeding. Manipulating whole-fly actin dynamics was achieved via feeding the flies either jasplakinolide (JPK) or latrunculin A (Lat.A). JPK and Lat.A were dissolved in 100\% DMSO. Flies were food deprived for $16 \mathrm{~h}$ before feeding on $250 \mathrm{~mm}$ liquid sucrose with either $200 \mathrm{nM} \mathrm{JPK}, 18.9$ $\mu \mathrm{M}$ Lat.A, or equivalent volume of DMSO vehicle. For the $M B-$ GeneSwitch experiments, flies were food deprived for $16 \mathrm{~h}$ before feeding on $250 \mathrm{~mm}$ liquid sucrose with or without $0.5 \mathrm{~mm}$ mifepristone (aka RU-486). A 50 mu mifepristone stock was dissolved in $95 \%$ ethanol and diluted for feeding. The resulting sucrose feeding solution including $1 \%$ ethanol was not sufficient to induce subsequent alcohol preference by itself (peer-reviewed data, data not shown).

Ethanol pre-exposures. Groups of 10 males were exposed to ethanol vapor and air via the Booze-O-Mat assay described previously (Wolf et al., 2002). Twenty minute exposures occurred $24 \mathrm{~h}$ before capillary feeder
(CAFÉ) assays. Control flies were mock-exposed to air vapor without ethanol.

Sixteen hour CAFÉ assay. Ethanol preference was performed using the two-bottle choice CAFÉ assay as described previously (Devineni and Heberlein, 2009) with modifications. Our CAFÉ assay consisted of a 6-well plate with four small holes drilled for the insertion of pipette tips and $20 \mu \mathrm{l}$ capillaries (VWR). Capillaries were filled via capillary action, and a small, black-stained mineral oil overlay was added to reduce evaporation. Preference assays with 10 males per well were conducted at $25^{\circ} \mathrm{C}$ and $70 \%$ relative humidity and flies chose between liquid sucrose food with or without $15 \%$ ethanol. For the MB-GeneSwitch experiments, food-deprived flies were fed $0.5 \mathrm{~mm}$ mifepristone for $3 \mathrm{~h}$ before the CAFÉ assay. Consumption data were scored based on the change in position of the mineral-oil dye marker overlaid on top of the liquid food in each capillary.

Sixteen hour COLA assay. The COLA (for CAFÉ-based online learning assay) apparatus was based on the described CAFÉ assay, but 4-well plates were used with two capillaries per well. The solutions offered were 250 mu sucrose with or without $15 \%$ ethanol. COLA assays were recorded with a time-lapse camera set to a 5 min interval (TLC200, Brinno). Video recordings were then binned into $2 \mathrm{~h}$ intervals to calculate preference for each interval. Consumption data were manually scored for every $2 \mathrm{~h}$ interval in the movie stills, based on the change in position of the mineral oil dye marker overlaid on top of the liquid food in each capillary.

Thirty minute FRAPPE assay. Naive alcohol aversion was tested in a 30 min two-choice preference assay called the FRAPPE (for fluorometric reading assay of preference primed by ethanol). This assay was performed as previously described (Peru y Colón de Portugal et al., 2014). Groups of 35 male flies chose between 340 mm liquid sucrose food with or without $15 \%$ ethanol, after a $6 \mathrm{~h}$ food deprivation.

$\mathrm{G} / \mathrm{F}$-actin in vivo assay. G/F-actin assays were performed according to the manufacturer's instructions (G/F-actin In Vivo Assay Kit, Cytoskeleton). G- and F-actin bands on Western blots were stained with antiactin (RRID:AB_10708070), and films were scanned by densitometry and the ratios of free $\mathrm{G}$-actin to F-actin were calculated.

Experimental design and statistical tests. Analysis of the experiments was performed using Prism 8 (GraphPad Software). The data were tested for normality by examining the QQ plots and using the Wilks-Shapiro normality test, which showed that the data were normally distributed with the exception of Figure 5. Data from the FRAPPE experiments were not normally distributed, as we previously found (Peru y Colón de Portugal et al., 2014), therefore these data were analyzed with nonparametric statistics. Data with an $n>8$ were checked for outliers, defined as $>2.5$ SDs outside the mean for single point measures (Figs. 1-4, 5 of 575 data points were excluded), and $1.5 \times$ the interquartile range outside the upper and lower quartiles for nonparametric data (Fig. 5, 3 of 332 data points excluded). For repeat measures, we excluded runs where more than half the time points lay $>2$ SD outside the mean (Fig. 6, 1 of 47 runs excluded). Post hoc analyses were corrected for multiple comparisons according to the Dunnett's method, when comparing numerous experimentals to one control, or Sidak's, when comparing predetermined pairs of means. Differences between SDs were tested with the Brown-Forsythe test, and no significant differences were found.

\section{Results}

\section{F-actin polymerization is involved in experience-dependent alcohol preference}

Our previous studies showed that wild-type flies naively avoid alcohol and choose to consume a sucrose solution over a sucrose solution containing alcohol in a two-choice paradigm (Peru y Colón de Portugal et al., 2014). This alcohol avoidance changes to preferential alcohol consumption over multiple days (Devineni and Heberlein, 2009) or with a pre-exposure to alcohol vapor the day before (Peru y Colón de Portugal et al., 2014). Here we recapitulate that result, showing that wild-type flies show slight naive avoidance to alcohol, but this avoidance switches to preference in a dose-dependent manner with the level of alcohol pre-exposure $24 \mathrm{~h}$ before the start of a $16 \mathrm{~h}$ abbreviated CAFÉ assay (Fig. 1B; 

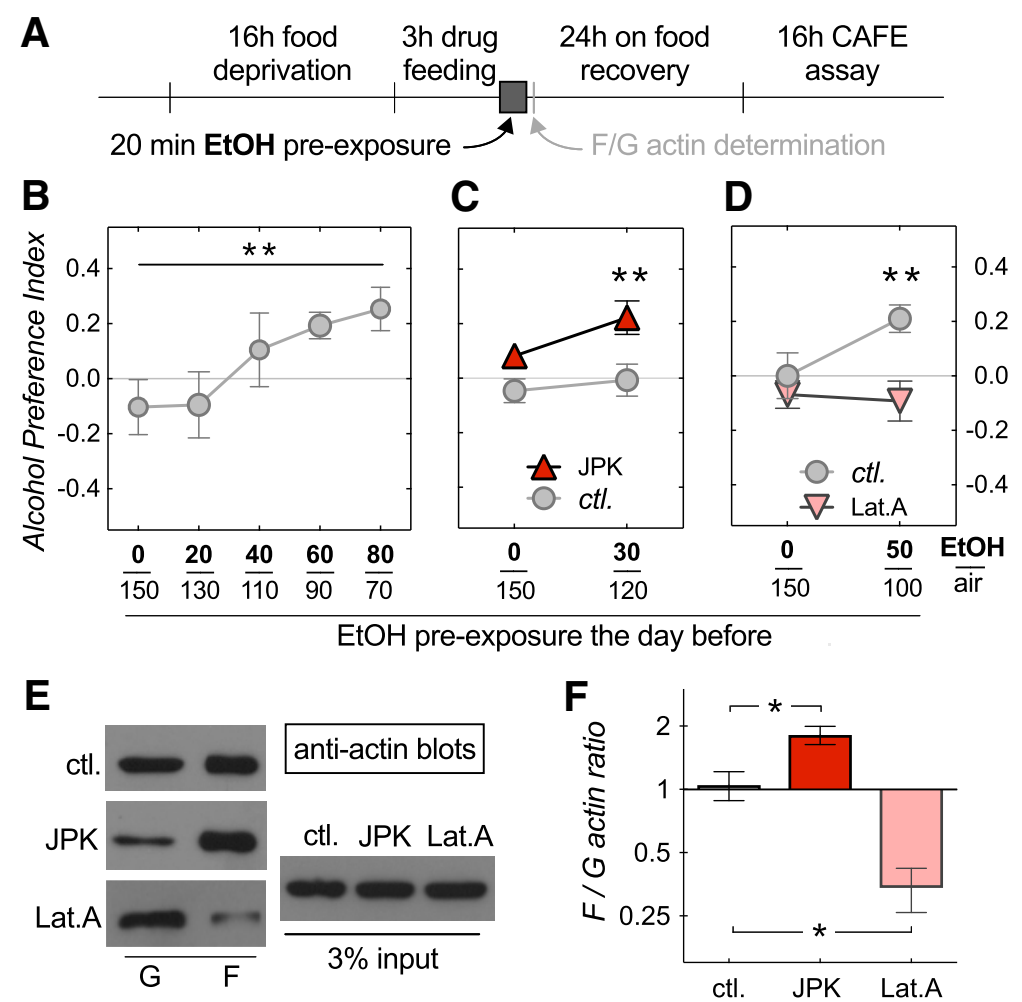

Figure 1. Effects of altered actin polymerization on EDAP. A, Schematic of the experimental design. Flies were food deprived for $16 \mathrm{~h}$ before a $3 \mathrm{~h}$ feeding on control food or food with either $200 \mathrm{~nm} \mathrm{JPK}$ to increase, or with $8.0 \mu \mathrm{g} / \mathrm{ml}$ Lat.A to decrease the $\mathrm{F} / \mathrm{G}$-actin ratio. Following the drug feeding, flies were pre-exposed to an ethanol vapor (EtOH) and air mixture for $20 \mathrm{~min}$, and then allowed to recover for $24 \mathrm{~h}$ on standard fly food. Flies were then assayed in a $16 \mathrm{~h}$ abbreviated CAFÉ assay. B, Control flies ( $w^{*}$ Berlin) show a dose-dependent switch from alcohol avoidance to alcohol preference (slope of linear regression $0.005 \pm 0.00395 \% \mathrm{Cl}$, significantly different from $0,{ }^{* *} p=0.0028, n=6$ per dose). Ethanol vapor to air ratios for the pre-exposures are displayed on the $x$-axis. C, Pre-feeding on JPK caused EDAP at a subthreshold pre-exposure dose (two-way ANOVA, with a significant effect: $F_{(1,44)}=11.9, p=0.0012$ of JPK; Sidak's multiple post hoc comparison, ${ }^{* *} p=0.006$ at $30 / 120 \mathrm{Et} 0 \mathrm{H} /$ air, $n=12$ per data point). $D$, Pre-feeding on Lat.A prevented EDAP at a dose which induced preference in control flies (two-way ANOVA, with a significant effect: $F_{(1,44)}=7.9, p=0.0074$ of Lat.A; Sidak's multiple post hoc comparison, ${ }^{* *} p=0.0046$ at 50/100 Et0H/air, $\left.n=12\right) . \boldsymbol{E}$, Anti-actin Western blots run from whole fly extracts. Blots on left show globular $(G)$ and filamentous $(F)$ actin fractions. The input extract shown on the right. $\boldsymbol{F}$, Western blot quantification shows significant, predicted changes in $\mathrm{F} / \mathrm{G}$-actin ratios (one-way ANOVA, $F_{(2,6)}=24.5, p=0.0013$, with Dunnett's post hoc multiple comparisons, ${ }^{*} p<0.027, n=3$; shown in this, and following figures, except Fig. 5, are mean with SE).

slope of linear regression $0.005 \pm 0.00395 \%$ CI, significantly different from $0,{ }^{*} p=0.0028, n=6$ per dose). Studies from our laboratory showed that manipulating regulators of actin dynamics, including Racl GTPase, can lead to behaviorally distinct alcohol phenotypes, including alcohol-induced sedation and locomotion activation (Rothenfluh et al., 2006; Ojelade et al., 2015a,b). Here, we wanted to investigate whether the manipulation of the state of F-actin would affect preferential alcohol consumption. Pre-feeding wild-type flies $200 \mathrm{~nm}$ JPK, a peptide with actin polymerization activity (Fig. $1 E, F$; one-way ANOVA, $F_{(2,6)}=24.5, p=0.0013$, with Dunnett's post hoc multiple comparisons, ${ }^{*} p<0.027, n=3$ ), was able to facilitate EDAP at a subthreshold pre-exposure dose of ethanol that did not induce preference in control flies (Fig. 1C; two-way ANOVA, with a significant effect, $F_{(1,44)}=11.9, p=0.0012$ of JPK. Sidak's multiple post hoc comparison ${ }^{*} p=0.006$ at $30 / 120 \mathrm{EtOH} /$ air, $n=12$ per data point). Conversely, pre-feeding flies $18.9 \mu \mathrm{M}$ Lat.A, a toxin that disrupts actin filaments and increases G-actin (Fig. $1 E, F)$, prevented the development of EDAP after alcohol preexposure at a higher dose (Fig. 1D; two-way ANOVA, with a significant effect, $F_{(1,44)}=7.9, p=0.0074$ of Lat.A. Sidak's mul- tiple post hoc comparison ${ }^{* *} p=0.0046$ at $50 / 100 \mathrm{EtOH} /$ air, $n=12$ ). These experiments show that feeding flies drugs interfering with F-actin polymerization before alcohol vapor pre-exposure alters their alcohol preference.

We next wanted to investigate whether proper regulation of actin dynamics is required during the $20 \mathrm{~min}$ alcohol preexposure, or during the $16 \mathrm{~h}$ preference test for normal EDAP development. Feeding flies Lat.A after the alcohol preexposure still prevented EDAP when tested 21 or $45 \mathrm{~h}$ after the drug feeding (Fig. 2D; two-way ANOVA, with a significant interaction: $F_{(1,20)}=2.3, p=0.021$; Sidak's multiple post hoc comparison, ${ }^{*} p=0.033$ at $50 / 100 \mathrm{EtOH} /$ air, $n=6 ; 2 E$, two-way ANOVA, with a significant interaction: $F_{(1,20)}=8.8, p=0.0075$; Sidak's multiple post hoc comparison, ${ }^{* *} p=$ 0.0094 at 50/100 EtOH/air, $n=6$ ). The effects of JPK, however, were more complicated: JPK-feeding no longer facilitated EDAP upon subthreshold alcohol preexposure, except for one time point, where it resulted in naive alcohol preference without alcohol pre-exposure (Fig. $2 B$; two-way ANOVA, with a significant effect: $F_{(1,32)}=4.8, p=0.037$, of JPK; Sidak's multiple post hoc comparison, ${ }^{*} p=$ 0.018 at $0 / 150 \mathrm{EtOH} / \mathrm{air}, n=6,12$ per data point; $2 C$ ). These data confirmed the importance of proper actin dynamics for EDAP, but its requirement during or after the pre-exposure was harder to interpret given the mixed results we obtained with JPK versus Lat.A. Furthermore, our feeding experiments were systemic, therefore complicating interpretation in light of pleiotropic alcohol phenotypes that can be obtained depending on which neurons are affected (Rothenfluh et al., 2006; Ojelade et al., 2015b). We did, however, test whether drug feeding altered flies' perception of ethanol, which might explain why Lat.A-fed flies never "acquired the taste" for alcohol (Fig. $1 D, 2 D, E$ ). We found that neither Lat.A nor JPK feeding changed naive alcohol aversion (see Fig. 5C).

\section{Increased mushroom body F-actin stability during pre- exposure suppresses EDAP}

Previously, we have shown that Ras suppressor 1 (Rsul) is a negative regulator of the small GTPase Racl. Rsul suppresses GTP loading of Rac1 and leads to an increase in F-actin (Ojelade et al., 2015b) via inactivation of the actin-severing protein cofilin (Ojelade et al., 2015a). We used three manipulations known to increase F-actin: UAS-Rsu1-RNAi to knock down Rsu1, UAS$R a c^{C A}$, to overexpress activated Rac1, and UAS-tsr ${ }^{D N}$ to overexpress dominant-negative, inactive cofilin. We previously showed that Rsul and Racl are required in the mushroom bodies (MB), a known center for associative learning (Cognigni et al., 2018), to develop alcohol preference in a $4 \mathrm{~d}$ CAFÉ assay (Ojelade et al., 2015b). We therefore restricted the genetic manipulations to the 

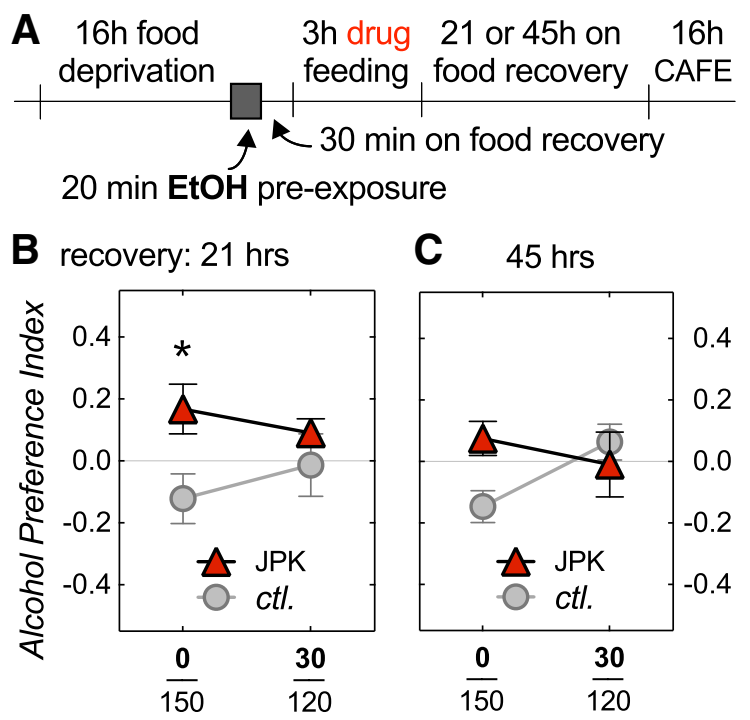

C $45 \mathrm{hrs}$

EtOH / air pre-exposure

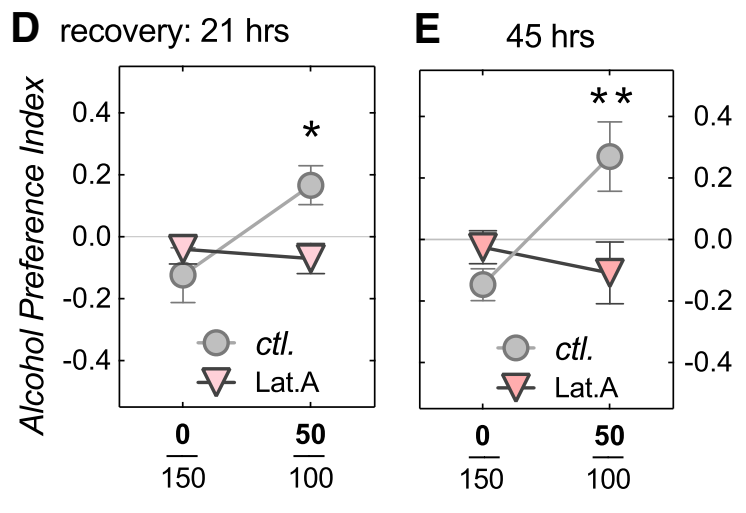

EtOH / air pre-exposure

Figure 2. Effects of actin polymerization post-acquisition on EDAP. A, Experimental design similar to Figure 1 with the exception that control, $w^{*}$ Berlin flies were drug-fed after alcohol vapor pre-exposure, and that two recovery times were tested. $\boldsymbol{B}, \boldsymbol{C}$, Feeding JPK after alcohol exposure led to naive preference in mock ethanol-exposed flies $21 \mathrm{~h}$ (two-way ANOVA, with a significant effect: $F_{(1,32)}=4.8, p=0.037$, of JPK; Sidak's multiple post hoc comparison, ${ }^{*} p=$ 0.018 at $0 / 150 \mathrm{Et} 0 \mathrm{H} / \mathrm{air}, n=6,12$ per data point), but not $45 \mathrm{~h}$ post-feeding. Ethanol-exposed flies showed no effect of JPK at either recover time. $\boldsymbol{D}, \boldsymbol{E}$, Feeding Lat.A after alcohol exposure prevented EDAP in ethanol-exposed flies $21 \mathrm{~h}$ (two-way ANOVA, with a significant interaction: $F_{(1,20)}=2.3, p=0.021$; Sidak's multiple post hoc comparison, ${ }^{*} p=0.033$ at $50 / 100 \mathrm{Et} 0 \mathrm{H} / \mathrm{air}$, $n=6$ ), and $45 \mathrm{~h}$ post-feeding (two-way ANOVA, with a significant interaction: $F_{(1,20)}=8.8$, $p=0.0075$; Sidak's multiple post hoc comparison, ${ }^{* *} p=0.0094$ at 50/100 Et0H/air, $n=6$ ).

MB using the drug-inducible Gal4, MB-GeneSwitch (MB-GS), which is activated upon consumption of mifepristone (aka RU486; Fig. $3 A, B)$. First, we activated $M B-G S$ before alcohol pre-exposure, which increases F-actin before acquisition of an alcohol preference (Fig. 3B). As expected, naive flies avoided alcohol in the $16 \mathrm{~h}$ CAFÉ (Fig. 3C). Following exposure to a preference-inducing dose of alcohol, F-actin mutants failed to develop preference, whereas the control, $M B-G S>U A S-G F P$, developed EDAP (Fig. 3D; one-way ANOVA, $F_{(3,73)}=15.0, p<$ 0.0001; Dunnett's multiple post hoc comparison ${ }^{* * *} p \leq 0.0008$, $n=12-18$, and 29 for GFP control), consistent with our previous findings (Ojelade et al., 2015b). To determine whether reduced F-actin dynamics, and increased F-actin, had a role after the acquisition of preference, we activated $M B-G S$ after the alcohol pre-exposure (Fig. 3E). As before, naive flies avoided alcohol in

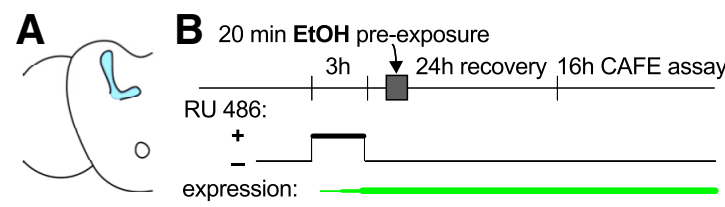

C pre-exposure: $\mathbf{0} / 150$

D $\quad 50 / 100$

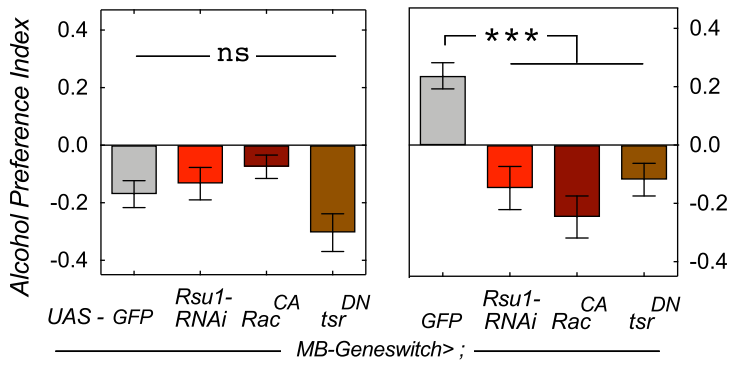

E 20 min EtOH pre-exposure
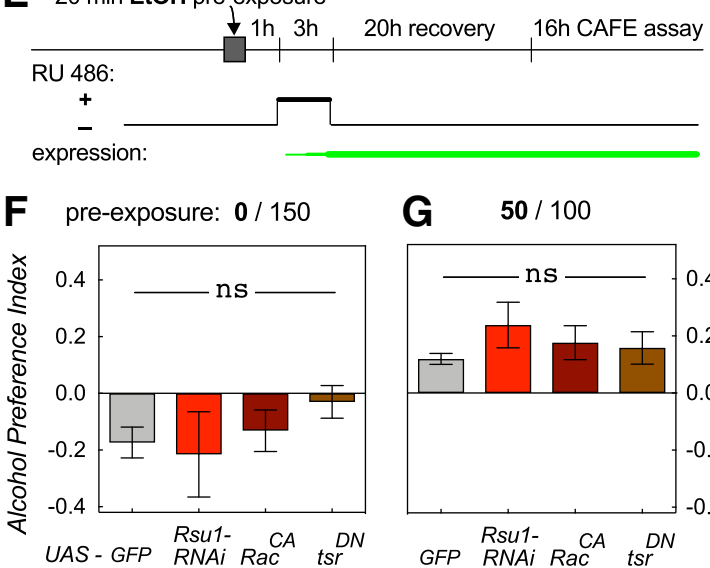

G $\quad 50 / 100$

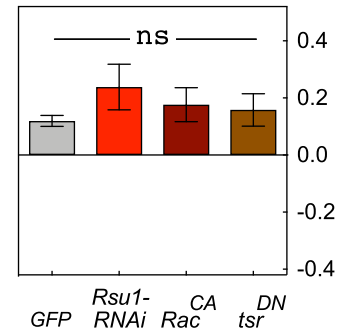

Figure 3. Decreasing F-actin turnover in the MB during pre-exposure suppresses EDAP. $\boldsymbol{A}$, Brain schematic highlighting the MB. $B$, Experimental design with RU486 feeding before ethanol exposure to activate the MB-GeneSwitch Gal4 driver. $C$, Decreasing F-actin turnover via three different transgenes did not alter naive alcohol avoidance (one-way ANOVA, $F_{(3,56)}=2.6, p=$ 0.06 , ns, not significant, $n=12 ; 24$ for GFP control). $D$, Decreasing F-actin turnover with transgene activation before ethanol pre-exposure prevented EDAP (one-way ANOVA, $F_{(3,73)}=$ $15.0, p<0.0001$; Dunnett's multiple post hoc comparison ${ }^{* *} p \leq 0.0008, n=12-18$; 29 for GFP control). $\boldsymbol{E}-\boldsymbol{G}$, Activating MB-GeneSwitch after alcohol pre-exposure had no effect on naive avoidance (one-way ANOVA, $F_{(3,32)}=0.7, p=0.55, n=6 ; 18$ for GFP control; $\boldsymbol{F}$ ), or on EDAP (one-way ANOVA, $F_{(3,32)}=1.4, p=0.27, n=6 ; 17$ for GFP control; $\boldsymbol{G}$ ).

the 16 h CAFÉ (Fig. $3 F$ ). In contrast, all the F-actin mutants and control flies developed preference after alcohol pre-exposure (Fig. $3 G$; one-way ANOVA, $F_{(3,32)}=1.4, p=0.26, n=6$, or 17 for GFP control). Together, these data indicate proper actin dynamics is required during alcohol pre-exposure for the acquisition of EDAP.

F-actin turnover in the mushroom bodies produces naive preference for alcohol

We next investigated the effect of increased F-actin turnover on EDAP. To that end we expressed two transgenes in the MB using $M B-G S$. The first was a dominant-negative mutation of Rac1, which keeps Racl bound to GDP and inactive. The second manipulation was a constitutively active mutation in $t s r$ producing an activated cofilin protein. As before, these manipulations were restricted to the $\mathrm{MB}$ using $M B-G S$. Activating expression of $M B-G S$ before, or after alcohol pre-exposure did not disrupt $\mathrm{EDAP}$, and in the case of $\mathrm{Rac}^{\mathrm{DN}}$ even caused an increase in pref- 


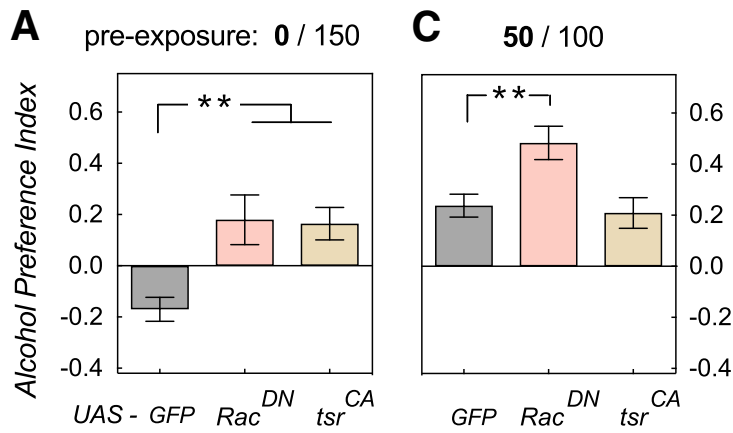

(activation before ethanol pre-exposure)

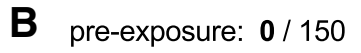

D $50 / 100$

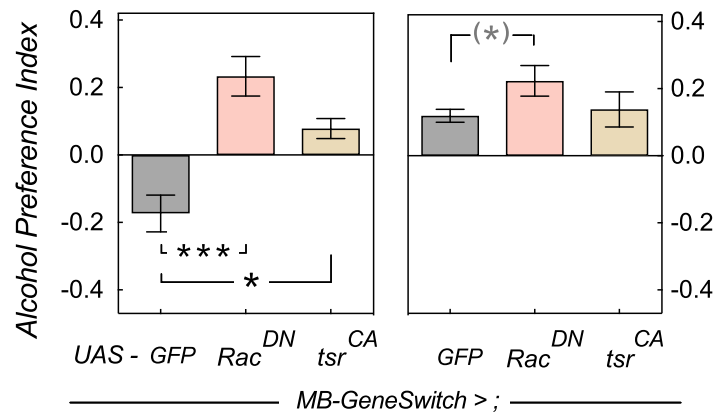

(activation after ethanol pre-exposure)

Figure 4. Increasing F-actin turnover in the MB leads to naive alcohol preference. $\boldsymbol{A}, \boldsymbol{B}$, Increasing $\mathrm{F}$-actin turnover with two different transgenes led to naive preference in mockexposed flies regardless of $M B$-GeneSwitch activation before (one-way ANOVA, $F_{(2,45)}=10.6$, $p=0.0002$; Dunnett's multiple post hoc comparison ** $p<0.0013, n=12 ; 24$ for GFP control; A) or after alcohol pre-exposure (one-way ANOVA, $F_{(2,27)}=11.2, p=0.0003$; Dunnett's multiple post hoc comparison ${ }^{* * *} p=0.0003,{ }^{*} p=0.021, n=6 ; 18$ for GFP control; $\left.\boldsymbol{B}\right)$. $\boldsymbol{C}$, Increasing $\mathrm{F}$-actin turnover before ethanol pre-exposure in alcohol pre-exposed flies still led to EDAP, and in the case of Rac ${ }^{\mathrm{DN}}$, enhanced preference (one-way ANOVA, $F_{(2,56)}=6.7, p=$ 0.0025 ; Dunnett's multiple post hoc comparison ${ }^{* *} p=0.0031, n=12,18$; 29 for GFP control). $D$, Activating MB-GeneSwitch after pre-exposure also still allowed development of EDAP. Oneway ANOVA indicated no differences $\left(F_{(2,27)}=2.8, p=0.075\right)$, whereas a $t$ test with Dunnett's correction suggested significant enhancement of EDAP with $\operatorname{Rac}^{\mathrm{DN}}\left(q=2.4, \mathrm{df}=29,{ }^{*} p=\right.$ 0.049 , effect size: Hedge's $g=0.95,0.11-1.8095 \%(\mathrm{Cl}, n=9,17$, and 6 for $t s r)$.

erence (Fig. 4C; one-way ANOVA, $F_{(2,56)}=6.7, p=0.0025$; Dunnett's multiple post hoc comparison, ${ }^{* *} p=0.0031, n=12,18$ or 29 for GFP control; $4 D$, one-way ANOVA indicated no differences, $\left.F_{(2,27)}=2.8, p=0.075\right)$. Surprisingly, overexpression of these mutants also caused preference in naive flies, regardless of whether we induced $M B$ - $G S$ activation before (Fig. $4 A$; one-way ANOVA, $F_{(2,45)}=10.6, p=0.0002$; Dunnett's multiple post hoc comparison ${ }^{* *} p<0.0013, n=12$, or 24 for GFP control) or after alcohol exposure (Fig. $4 B$; one-way ANOVA, $F_{(2,27)}=11.2, p=$ 0.0003; Dunnett's multiple post hoc comparison, ${ }^{\star *} p=0.0003$, ${ }^{*} p=0.021, n=6$, or 18 for GFP control). The transgenes' effects were very strong, with significant effect sizes (Hedge's $g$ ) between 1.2 and 1.8 (Fig. 4A,B). This was unexpected for two reasons. First our results with the opposite mutations (Fig. 3) suggested that actin dynamics during, not after, the pre-exposure are critical for preference development. Second, and even more surprising, our prior findings suggested that the $\mathrm{MB}$ are critical for EDAP, but that neurons outside the MB are involved in mediating and determining naive alcohol avoidance (Ojelade et al., 2015b). This led us to two alternative hypotheses; the first that increased F-actin turnover in the MB does in fact affect naive alcohol avoidance. The second hypothesis was that enhanced
F-actin turnover gradually increases the voluntary consumption of alcohol compared with controls specifically during our $16 \mathrm{~h}$ "testing" period.

\section{Increased F-actin turnover mutants display normal naive alcohol aversion}

To investigate our first hypothesis, we assayed our increased F-actin turnover mutants in a short 30 min two-choice preference assay, called FRAPPE. This assay pairs food offerings with a fluorometric dye, which can be quantified through the cuticle of the fly abdomen to determine which food was preferred (Peru y Colón de Portugal et al., 2014). Flies with adult-induced expression of $M B-G S>U A S-R a c^{D N}$ showed no difference in naive alcohol aversion compared with control flies (Fig. 5A; Kruskal-Wallis test, statistic $=2.09, p=0.35, n=47$ or 48$)$. Because the timing of expression of the mutants had no impact on the phenotype in Figure 4, we also used a constitutive MB-specific driver, MB247Gal4. As seen with $\mathrm{Rac}^{\mathrm{DN}}, M B 247-G a l 4>U A S-t_{s}{ }^{C A}$ flies also displayed naive alcohol aversion in our $30 \mathrm{~min}$ preference assay, as did the control flies (Fig. 5B; Mann-Whitney test, $U=216, p=$ $0.86, n=14,32$ ). Last, we also fed control flies Lat.A and JPK, to alter actin dynamics, and neither drug caused a change in naive alcohol aversion (Fig. 5C; Kruskal-Wallis test, statistic $=1.46$, $p=0.48, n=45-48)$. Therefore, reduced naive alcohol aversion did not explain why above transgenic mutants showed preference in our $16 \mathrm{~h}$ CAFÉ assay in the absence of prior ethanol exposure (Fig. $4 A, B$ ) or when fed JPK after the ethanol pre-exposure (Fig. $2 B)$.

\section{Increased F-actin turnover in the MB accelerates the acquisition of alcohol preference}

We designed our $16 \mathrm{~h} \mathrm{CAFÉ} \mathrm{assay} \mathrm{as} \mathrm{the} \mathrm{preference} \mathrm{testing} \mathrm{phase}$ of our EDAP paradigm. However, our above results suggested that mutants with increased F-actin turnover might start out avoiding alcohol, but then acquire preference within this $16 \mathrm{~h}$ testing period. To investigate this idea, we had to develop an assay that could monitor preference in real time, as opposed to checking consumption and preference at the end of the $16 \mathrm{~h}$ testing period, or every $24 \mathrm{~h}$ as for long-term CAFÉ assays (Ja et al., 2007; Devineni and Heberlein, 2009). We therefore developed the CAFÉ-based online learning assay (COLA), which differs from a traditional CAFÉ because consumption of the food offerings is video recorded in real time, allowing minute-level resolution of feeding behavior. Because feeding in the COLA is sporadic, and not continuous, we decided to check preference and consumption every $2 \mathrm{~h}$. Flies with induced $M B-G S>\operatorname{Rac}^{D N}$ showed a gradual increase of the preference index (PI) over the $16 \mathrm{~h}$ experiment, which was less evident for the un-induced control (Fig. 6A; twoway RM ANOVA for repeat measures, $F_{(2.118,42.37)}=7.6,{ }^{\star *} p=$ 0.0012 significant effect of time and of interaction: time $\times$ RU486 treatment, $\left.F_{(7,140)}=3.1,{ }^{*} p=0.0042, n=11\right)$. When we calculated an interval PI for the first and last $6 \mathrm{~h}$ of the experiment, experimental $\operatorname{Rac}^{\mathrm{DN}}$ flies showed a significantly higher PI at the end of the experiment than controls, showing that they acquired a preference increase for the duration of the $16 \mathrm{~h}$ experiment (Fig. $6 B$; one-way ANOVA with 2 pre-selected Sidak's pairwise comparisons, $\left.t=3.17,{ }^{* *} p=0.0058\right)$. Because the PI change was most obvious in the first $8 \mathrm{~h}$, we also determined the slope of a linear regression curve through the first four $2 \mathrm{~h}$ PI intervals for each replicate, essentially asking whether there is an increase in alcohol preference within the first $8 \mathrm{~h}$ of the experiment. The average slope of these linear regressions was significantly positive for the induced experimental $\operatorname{Rac}^{\mathrm{DN}}$ flies, but not for the un-induced 
controls (Fig. 6C; one sample $t$ test with Bonferroni threshold adjustment, $t=$ $1.91,{ }^{\mathrm{ns}} p=0.085$ for - group, $t=3.18$, ${ }^{*} p=0.0098$ for + group). It is noteworthy that both control and experimental flies showed a fairly high, albeit indistinguishable, preference even within the first $2 \mathrm{~h}$ of the experiment. There are two reasons for that: first, flies show more alcohol aversion in our 30 min assay (Fig. 5), which offers the food in numerous wells, and not small capillaries like in the CAFÉ. This is partially because of their aversion of the (relatively undiluted) smell of alcohol (data not shown). The other reason was the genetic background of the $M B-G S$ driver, which showed relatively high initial preference in the COLA setup, regardless of RU486 induction or UAS-transgene presence. We therefore switched to the MB247-Gal4 driver to test the effect of activated cofilin mutants. Experimental $M B 247>t s r^{C A}$ again showed increased preference over the $16 \mathrm{~h}$ experiment compared with controls (Fig. $6 D$; two-way RM ANOVA, $F_{(1.180,40.70)}=7.8,{ }^{\star *} p=0.0017$ for time; and $F_{(1,22)}=10.4,{ }^{* *} p=0.0039$ for genotype; and $\left(F_{(7,154)}=2.95,{ }^{* *} p=\right.$ 0.0062 for the genotype $\times$ time interaction). The preference at numerous time points was significantly increased with UAS-tsr ${ }^{C A}$ (Fig. 6D; Sidak's multiple comparisons, $t>3.3,{ }^{*} p<0.027$ ), and their PI was significantly higher than the control PI during both the first (Fig. 6E; oneway ANOVA with 2 pre-selected Sidak's pairwise comparisons, $t=2.81$, ${ }^{*} p=$ $0.0148)$ and the last $6 \mathrm{~h}$ of the assay $(t=$ $\left.2.37,{ }^{*} p=0.0436\right)$. Last, experimental flies' average slope for the linearlyregressed first four $2 \mathrm{~h}$ interval PIs was also positive while the control's was not (Fig. 6F; one-sample $t$ test with Bonferroni threshold adjustment, $t=0.97,{ }^{\mathrm{ns}} p=$ 0.35 for GFP group, $t=3.31,{ }^{*} p=0.0069$ for $t s r^{C A}$ group), indicating that $M B 247>t s r^{C A}$ flies learn to prefer alcohol within the first $8 \mathrm{~h}$ of the assay (also visually evident in Fig. $6 D)$.

\section{Discussion}

Requirement for mushroom body F-actin turnover in the development of alcohol preference

Proper dynamic regulation of the actin cytoskeleton is known to be relevant for learning and memory (Lamprecht, 2016), including the development of drug preference (Rothenfluh and Cowan, 2013). Our systemic drug-feeding experiments in Figure 1 suggested that increasing F-actin facilitates the development of EDAP, while decreasing F-actin abolished preference. However, when we restricted the manipulations of F-actin to the

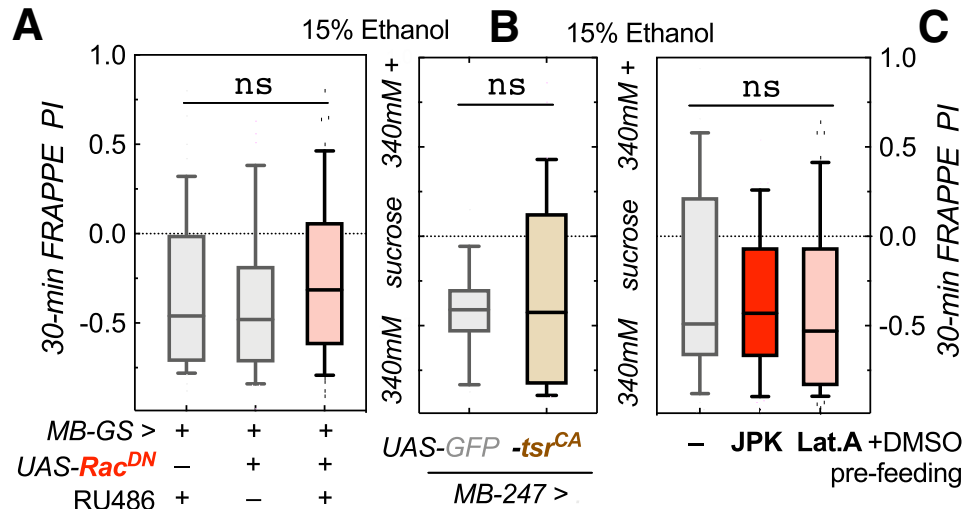

Figure 5. Increased MB F-actin turnover does not affect naive alcohol avoidance in a short preference assay. $A$, Adult expression of UAS-RaC ${ }^{D N}$ with MB-GeneSwitch did not alter avoidance in a 30 min preference assay (Kruskal-Wallis test, statistic $=2.09, p=0.35, \mathrm{~ns}$, not significant, $n=47$ or 48 ; shown are medians with quartile boxes and $10-90 \%$ whiskers). $B$, Constitutive UAS-tsr ${ }^{C A}$ expression with MB247-Gal4 did not change naive alcohol avoidance either (Mann-Whitney test, $U=216, p=0.86, n=14,32$ ). C, Feeding $W^{*}$ Berlin control flies Lat.A or JPK did not change their naive alcohol avoidance (Kruskal-Wallis test, statistic $=1.46, p=0.48, n=45-48$ ).

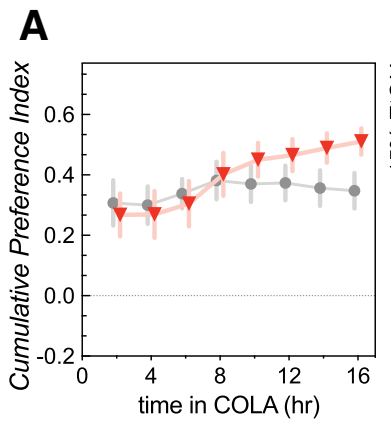

D

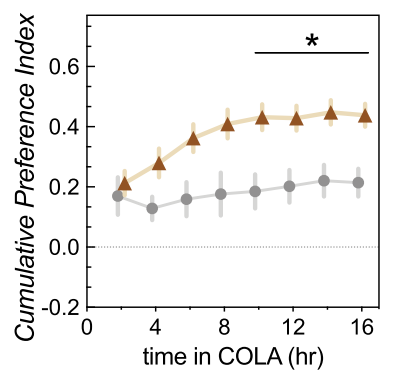

B

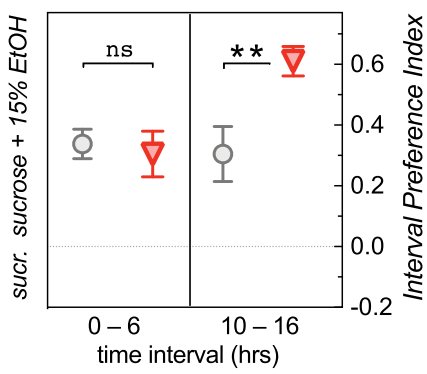

E
C

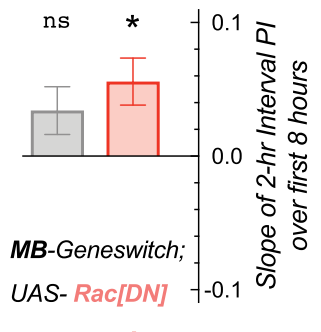

$+\quad+\mathrm{RU} 486$

$F$

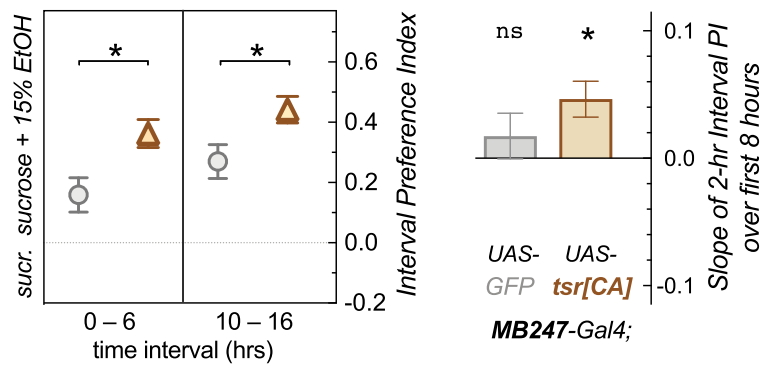

Figure 6. Increasing F-actin turnover in the MB causes fast acquisition of alcohol preference. Experiments were conducted in a CAFÉ online learning assay, which monitored consumption from the food capillaries with 5 min resolution. $A$, Cumulative preference index, from time 0 to time $X$, for adult-expressed UAS-Rac ${ }^{D N}$ in the MB showed a significant effect of time (two-way RM ANOVA for repeat measures, $\left.F_{(2.118,42.37)}=7.6, p=0.0012\right)$ and of interaction (time $\times$ RU486 treatment: $F_{(7,140)}=3.1, p=0.0042$, $n=11$ ), but no post hoc differences (Sidak's multiple comparisons, $t<2.3, p>0.27$ ). $\boldsymbol{B}$, Interval preference index for the first and last $6 \mathrm{~h}$ revealed no difference at the start (one-way ANOVA with 2 pre-selected Sidak's pairwise comparisons, $t=0.35, p=0.93$, ns, not significant,), but a significant increase for the treated group $\left(t=3.17,{ }^{* *} p=0.0058\right)$. C, Average slope of the linear regression for the $2 \mathrm{~h}$ interval preferences in the first $8 \mathrm{~h}$. The RU486-treated group showed a positive average slope (one-sample $t$ test with Bonferroni threshold adjustment, $t=1.91,{ }^{\mathrm{ns}} p=0.085$ for - group, $t=3.18,{ }^{*} p=0.0098$ for + group). $\boldsymbol{D}$, Cumulative preference index when expressing $M B 247>t s r^{C A}$ showed significant effects of time (tw0-way RM ANOVA, $F_{(1.180,40.70)}$ $=7.8, p=0.0017)$, treatment (UAS-transgene, $\left.F_{(1,22)}=10.4, p=0.0039\right)$ and interaction $\left(F_{(7,154)}=2.95, p=0.0062\right)$. The preference at numerous time points was significantly increased with UAS-tsr ${ }^{C A}$ (Sidak's multiple comparisons, $t>3.3,{ }^{*} p<$ 0.027). $\boldsymbol{E}$, Interval preference indices were also increased during both the first $6 \mathrm{~h}$ (one-way ANOVA with 2 pre-selected Sidak's pairwise comparisons, $\left.t=2.81,{ }^{*} p=0.0148\right)$ and the last $6 \mathrm{~h}\left(t=2.37,{ }^{*} p=0.0436\right)$. $\boldsymbol{F}$, The average slope of the linear regression in the first $8 \mathrm{~h}$ was positive for the experimental $t s r^{C}{ }^{C}$, but not GFP control group (one-sample $t$ test with Bonferroni threshold adjustment, $t=0.97,{ }^{\text {ns }} p=0.35$ for GFP group, $t=3.31,{ }^{*} p=0.0069$ for $t s r^{C A}$ group).

$\mathrm{MB}$, our data suggested that increased MB F-actin abolished EDAP, whereas decreased F-actin enhanced EDAP. This discrepancy may be explained by two possibilities. First, the MB are a well-known center for learning and memory in flies (Cognigni et 
al., 2018) and have been shown to be involved in alcohol consumption preference (Kaun et al., 2011; Xu et al., 2012). Conversely, we have shown that neurons outside the MB can also affect alcohol preference (Ojelade et al., 2015b). Therefore, altering F-actin systemically changes actin dynamics both in $\mathrm{MB}$ and other neurons, and the resulting behavioral output is the sum of the different circuits affecting behavioral responses. Thus, MBspecific changes might lead to a different phenotype than systemic changes. An alternative explanation would be the actual mechanism of action of Lat.A compared with cofilin, which both lead to decreased F-actin. Lat.A binds to G-actin monomers and prevents actual polymerization of actin filaments (Morton et al., 2000), whereas cofilin causes severing of actin filaments, but does not prevent new formation of filaments. Indeed, cofilin activity increases actin treadmilling and membrane protrusion (Kanellos and Frame, 2016). Thus, Lat.A may be viewed as decreasing overall dynamics of F-actin turnover, whereas activated cofilin increases $\mathrm{F}$-actin turnover and regeneration. The relevant change induced by these manipulations may not be in the overall F/Gactin ratio, but rather the dynamic ability for the F-actin cytoskeleton to change on demand. Indeed, the mechanism of action for JPK is not to just rigidly stabilize actin filaments, but also to enhance the rate of F-actin nucleation (Bubb et al., 2000). Together, these results suggest that the proper development of EDAP requires enhanced F-actin dynamics and turnover in the Drosophila MB, which is counteracted by $\mathrm{Rac}^{\mathrm{CA}}$ (Fig. 3D). Similarly, Dietz et al. (2012) found that overexpression of $\mathrm{Rac}^{\mathrm{CA}}$ in the rodent nucleus accumbens reduced cocaine-induced place preference, highlighting the importance of this GTPase in drug abuse across phyla.

\section{The temporal requirement of proper F-actin dynamics in preference development}

Our behavioral paradigm consists of an alcohol pre-exposure followed by a preference test. We were therefore able to ask whether proper F-actin dynamics is required during the preexposure and acquisition of preference, or at later stages, i.e., testing, or consolidation. Our data with reduced F-actin turnover (Fig. 3) suggested that enhanced F-actin dynamics is required during the acquisition of preference, but not thereafter. However, decreasing F-actin with systemic Lat.A feeding after the preexposure also led to abolished EDAP (Fig. 2D,E). Thus, some $\mathrm{F}$-actin generation, or at the very least, F-actin stabilization may also be required during the consolidation or recall phase of alcohol-induced consumption preference. Indeed, injection of Lat.A into the rodent basolateral amygdala after training and consolidation of methamphetamine-induced conditioned place preference (CPP) abolished the expression/recall of that place memory (Young et al., 2014), whereas injection of Lat.A into the nucleus accumbens shell led to reduced consolidation of morphine-induced CPP (Li et al., 2015). These data suggest that proper $\mathrm{F}$-actin dynamics is important during numerous stages of addiction-related behavior, including during the acquisition of drug-preference memory, as our data suggest.

\section{Fast acquisition of preferential alcohol self-administration with enhanced F-actin turnover}

Enhancing F-actin dynamics led to facilitated alcohol preference, even in the absence of an alcohol pre-exposure (Fig. 4A,B). Our subsequent analysis showed that this is not due to altered naive avoidance of alcohol (Fig. 5; Peru y Colón de Portugal et al., 2014), but rather because these flies showed fast acquisition of alcohol preference. We determined this using our novel COLA assay, which allows for much refined temporal resolution of monitoring self-administration. The parsimonious explanation for the fast acquisition would be that increased F-actin dynamics enhances the acquisition of the alcohol preference. Supporting this interpretation are findings that overexpression of dominantnegative Rac or activated cofilin in the nucleus accumbens was able to induce CPP with a subthreshold dose of cocaine (Dietz et al., 2012), suggesting that these molecular manipulations facilitate the acquisition of a drug-induced memory. In addition, Drosophila exposed to a weak olfactory conditioning paradigm showed low levels of long-term memory (Johnson et al., 2015). But when these flies were pre-fed with JPK and then weakly trained, they showed significantly enhanced levels of long-term memory (Johnson et al., 2015), supporting the notion that enhanced actin filament turnover facilitates memory acquisition.

A number of reports have suggested that activated Rac leads to active forgetting of shock-conditioned odor avoidance, whereas dominant-negative Rac and activated cofilin reduce the rate of forgetting (Shuai et al., 2010; Cervantes-Sandoval et al., 2016). Our results could be interpreted in a similar light, with $M B>R a c^{C A}$ flies not displaying EDAP (Fig. 3D), because they forgot it right away. Conversely, $M B>R a c^{D N}$ and $t s r^{C A}$ showed enhanced preference (Figs. 4,6), because they did not forget any of it. However, induction of Rac ${ }^{\mathrm{CA}}$ after ethanol pre-exposure did not affect EDAP (Fig. 3E), showing that activated Rac does not drive an active reduction/forgetting of the acquired ethanol-memory. $\mathrm{Rac}^{\mathrm{DN}}$ affected shock-induced odor memory up to $24 \mathrm{~h}$ after the memory induction (Shuai et al., 2010), thus it seems unlikely that the timing of $R a c^{C A}$ expression after the ethanol pre-exposure is the cause for the lack of a $\mathrm{Rac}^{\mathrm{CA}}$ effect on alcohol preference. The observed difference to shock-conditioned odor avoidance may simply be a reflection of the different memories, one stemming from aversive punishment, and the other from appetitive reinforcement. Even within appetitive conditioning, numerous reports suggest that drug-induced memories are stronger, or even categorically different from sucrose-induced ones. These drugmemories are unaffected by manipulations affecting F-actin that do affect food memories (Kiraly et al., 2010; Young et al., 2014; Laguesse et al., 2017). Regardless of the exact function of this pathway leading to activated cofilin and F-actin dynamics in suppressing forgetting and/or facilitating memory acquisition, we here show that this pathway can bidirectionally affect the development of alcohol self-administration preference. Our data also demonstrate that increased F-actin turnover in the Drosophila $\mathrm{MB}$ can facilitate the acquisition of drug memories, suggesting a high degree of evolutionary conservation of this pathway's function in the development of substance abuse disorders.

\section{References}

Bubb MR, Spector I, Beyer BB, Fosen KM (2000) Effects of jasplakinolide on the kinetics of actin polymerization. an explanation for certain in vivo observations. J Biol Chem 275:5163-5170.

Centers for Disease Control and Prevention (2014) Alcohol and public health: Alcohol-related disease impact (ARDI). Average for United States 2006-2010 alcohol-attributable deaths due to excessive alcohol use. Washington, DC.

Cervantes-Sandoval I, Chakraborty M, MacMullen C, Davis RL (2016) Scribble scaffolds a signalosome for active forgetting. Neuron 90:12301242.

Cognigni P, Felsenberg J, Waddell S (2018) Do the right thing: neural network mechanisms of memory formation, expression and update in Drosophila. Curr Opin Neurobiol 49:51-58.

Devineni AV, Heberlein U (2009) Preferential ethanol consumption in Drosophila models features of addiction. Curr Biol 19:2126-2132.

Dick DM, Jones K, Saccone N, Hinrichs A, Wang JC, Goate A, Bierut L, 
Almasy L, Schuckit M, Hesselbrock V, Tischfield J, Foroud T, Edenberg H, Porjesz B, Begleiter H (2006) Endophenotypes successfully lead to gene identification: results from the collaborative study on the genetics of alcoholism. Behav Genet 36:112-126.

Dietz, Sun H, Lobo MK, Cahill ME, Chadwick B, Gao V, Koo JW, MazeiRobison MS, Dias C, Maze I, Damez-Werno D, Dietz KC, Scobie KN, Ferguson D, Christoffel D, Ohnishi Y, Hodes GE, Zheng Y, Neve RL, Hahn KM, DM et al. (2012) Racl is essential in cocaine-induced structural plasticity of nucleus accumbens neurons. Nat Neurosci 15:891-896.

Evangelou, Gao H, Chu C, Ntritsos G, Blakeley P, Butts AR, Pazoki R, Suzuki H, Koskeridis F, Yiorkas AM, Karaman I, Elliott J, Luo Q, Aeschbacher S, Bartz TM, Baumeister SE, Braund PS, Brown MR, Brody JA, Clarke TK, E et al. (2019) New alcohol-related genes suggest shared genetic mechanisms with neuropsychiatric disorders. Nat Hum Behav 3:950-961.

Gelernter J, Kranzler HR (2010) Genetics of drug dependence. Dialogues Clin Neurosci 12:77-84.

Gonzalez DA, Jia T, Pinzón JH, Acevedo SF, Ojelade SA, Xu B, Tay N, Desrivières $S$, Hernandez JL, Banaschewski T, Büchel C, Bokde ALW, Conrod PJ, Flor H, Frouin V, Gallinat J, Garavan H, Gowland PA, Heinz A, Ittermann B, et al. (2018) The Arf6 activator Efa6/PSD3 confers regional specificity and modulates ethanol consumption in Drosophila and humans. Mol Psychiatry 23:621-628.

Grotewiel M, Bettinger JC (2015) Drosophila and Caenorhabditis elegans as discovery platforms for genes involved in human alcohol use disorder. Alcohol Clin Exp Res 39:1292-1311.

Ja WW, Carvalho GB, Mak EM, de la Rosa NN, Fang AY, Liong JC, Brummel T, Benzer S (2007) Prandiology of Drosophila and the CAFE assay. Proc Natl Acad Sci U S A 104:8253-8256.

Johnson JL, Huang W, Roman G, Costa-Mattioli M (2015) TORC2: a novel target for treating age-associated memory impairment. Sci Rep 5:15193.

Kanellos G, Frame MC (2016) Cellular functions of the ADF/cofilin family at a glance. J Cell Sci 129:3211-3218.

Kaun KR, Azanchi R, Maung Z, Hirsh J, Heberlein U (2011) A Drosophila model for alcohol reward. Nat Neurosci 14:612-619.

Kaun KR, Devineni AV, Heberlein U (2012) Drosophila melanogaster as a model to study drug addiction. Hum Genet 131:959-975.

Kiraly DD, Ma XM, Mazzone CM, Xin X, Mains RE, Eipper BA (2010) Behavioral and morphological responses to cocaine require Kalirin7. Biol Psychiatry 68:249-255.

Laguesse S, Morisot N, Shin JH, Liu F, Adrover MF, Sakhai SA, Lopez MF, Phamluong K, Griffin WC 3rd, Becker HC, Bender KJ, Alvarez VA, Ron D (2017) Prosapip1-dependent synaptic adaptations in the nucleus accumbens drive alcohol intake, seeking, and reward. Neuron 96:145-159.e8.

Lamprecht R (2016) The role of actin cytoskeleton in memory formation in amygdala. Front Mol Neurosci 9:23.

Lee HG, Kim YC, Dunning JS, Han KA (2008) Recurring ethanol exposure induces disinhibited courtship in Drosophila. PLoS One 3:e1391.

Li G, Wang Y, Yan M, Xu Y, Song X, Li Q, Zhang J, Ma H, Wu Y (2015)
Inhibition of actin polymerization in the NAc shell inhibits morphineinduced CPP by disrupting its reconsolidation. Sci Rep 5:16283.

Morton WM, Ayscough KR, McLaughlin PJ (2000) Latrunculin alters the actin-monomer subunit interface to prevent polymerization. Nat Cell Biol 2:376-378.

Ojelade SA, Acevedo SF, Kalahasti G, Rodan AR, Rothenfluh A (2015a) RhoGAP18B isoforms act on distinct rho-family GTPases and regulate behavioral responses to alcohol via cofilin. PLoS One 10:e0137465.

Ojelade SA, Jia T, Rodan AR, Chenyang T, Kadrmas JL, Cattrell A, Ruggeri B, Charoen P, Lemaitre H, Banaschewski T, Büchel C, Bokde AL, et al. (2015b) Rsul regulates ethanol consumption in Drosophila and humans. Proc Natl Acad Sci U S A 112:E4085-E4093.

Osterwalder T, Yoon KS, White BH, Keshishian H (2001) A conditional tissue-specific transgene expression system using inducible GAL4. Proc Natl Acad Sci U S A 98:12596-12601.

Peru y Colón de Portugal RL, Ojelade SA, Penninti PS, Dove RJ, Nye MJ, Acevedo SF, Lopez A, Rodan AR, Rothenfluh A (2014) Long-lasting, experience-dependent alcohol preference in Drosophila. Addict Biol 19:392-401.

Rodan AR, Rothenfluh A (2010) The genetics of behavioral alcohol responses in Drosophila. Int Rev Neurobiol 91:25-51.

Rothenfluh A, Cowan CW (2013) Emerging roles of actin cytoskeleton regulating enzymes in drug addiction: actin or reactin'? Curr Opin Neurobiol 23:507-512.

Rothenfluh A, Threlkeld RJ, Bainton RJ, Tsai LT, Lasek AW, Heberlein U (2006) Distinct behavioral responses to ethanol are regulated by alternate RhoGAP18B isoforms. Cell 127:199-211.

Sacks JJ, Gonzales KR, Bouchery EE, Tomedi LE, Brewer RD (2015) 2010 national and state costs of excessive alcohol consumption. Am J Prev Med 49:e73-e79.

Shuai Y, Lu B, Hu Y, Wang L, Sun K, Zhong Y (2010) Forgetting is regulated through Rac activity in Drosophila. Cell 140:579-589.

Substance Abuse and Mental Health Services Administration (2015) National survey on drug use and health. Table 2.41B and Table 5.6A. Rockville, $\mathrm{MD}$.

Wolf FW, Rodan AR, Tsai LT, Heberlein U (2002) High-resolution analysis of ethanol-induced locomotor stimulation in Drosophila. J Neurosci 22:11035-11044

Xu S, Chan T, Shah V, Zhang S, Pletcher SD, Roman G (2012) The propensity for consuming ethanol in Drosophila requires rutabaga adenylyl cyclase expression within mushroom body neurons. Genes Brain Behav 11:727-739.

Young EJ, Aceti M, Griggs EM, Fuchs RA, Zigmond Z, Rumbaugh G, Miller CA (2014) Selective, retrieval-independent disruption of methamphetamineassociated memory by actin depolymerization. Biol Psychiatry 75:96-104.

Zars T, Fischer M, Schulz R, Heisenberg M (2000) Localization of a shortterm memory in Drosophila. Science 288:672-675. 